\title{
Bilateral foot drop, weight loss and rectal bleeding as an acute presentation of Crohn's disease
}

\author{
S.E. Gariballa and N.P.R. Gunasekera \\ Department of General Medicine and Department of Medicine for the Elderly, Kettering General Hospital, \\ Rothwell Road, Kettering NN16 8UZ, UK
}

\begin{abstract}
Summary: We report a 71 year old lady who presented with weight loss, rectal bleeding and bilateral foot drop having been previously fit and well. Clinical examination, laboratory investigation and postmortem examination confirmed the diagnosis of active Crohn's disease and acute peripheral neuropathy. The clinical course of this patient suggests that the peripheral neuropathy might have resulted from the common pathogenesis for Crohn's disease.
\end{abstract}

\section{Introduction}

Crohn's disease has numerous extra-intestinal manifestations with notable sparing of the nervous system, especially the peripheral nerves. In this report we describe a patient who presented acutely with active Crohn's disease and peripheral neuropathy, a presentation that raises a question about the possible association of the two disorders.

\section{Case history}

A 71 year old woman, previously fit and independent, presented with a 2 week history of unsteadiness and lower limb weakness, and a one week history of rectal bleeding and loose motions 5-6 times/day. She also gave a history of weight loss of around one stone in the previous month. Prior to this she enjoyed good health apart from hypertension for a number of years for which she took atenolol, chlorthalidone, amlodipine $5 \mathrm{mg}$ / day and aspirin $75 \mathrm{mg}$ daily.

On general examination the only abnormal physical sign was bilateral foot drop with marked weakness of the dorsiflexors bilaterally; tendon reflexes in the lower limbs were present but sluggish. Proctosigmoidoscopy revealed normal colour stools but red inflamed mucosa. A rectal biopsy was taken that concern the diagnosis of acute Crohn's disease.

Other laboratory tests included ESR $82 \mathrm{~mm} /$ hour, C-reactive protein $168 \mathrm{mg} / 1$ (normal $<6 \mathrm{mg}$ / 1 , albumin $24 \mathrm{~g} / \mathrm{l}$, potassium $2.6 \mathrm{mmol} / 1$, haemo-

Correspondence: S.E. Gariballa, M.R.C.P., 39 Hospital Close, Leicester LE5 4WP, UK.

Accepted: 25 April 1994 globulin $11 \mathrm{~g} / \mathrm{dl}$. The remainder of the biochemistry was normal as were immunoglobulins, proteins, electrophoresis, $\mathbf{B}_{12}$ folate rheumatoid factor, antinuclear and anti-neutrophil cytoplasmic antibodies. Stool microscopy and culture were negative on three occasions. Barium enema and lumbar puncture were unsuccessful. Sural sensory studies showed small units with normal velocity suggestivece of an axonal type of neuropathy. Both lateral popliteal (common peroneal) nerve motor studies showed evoked response in extensor digitorum brevis stimulating at the ankles. More proximal studies were within normal limits except for an increased latency of $6.4 \mathrm{~ms}$ from the neck of fibula to the upper tibialis anterior on the left. Electromyography of the left extensor digitorum brevis showed no activity at rest and no units under voluntary control, whilst the left tibialis anterior showed no activity at rest and the pattern on maximum voluntary effort was reduced. Left posterior tibial nerve motor studies to the abductor hallucis were normal. In conclusion there were some changes of peripheral nerve dysfunction in both motor and sensory studies, with most changes in the distal lateral popliteal nerves.

As soon as the diagnosis of acute Crohn's disease had been made she was started on treatment with oral prednisolone $30 \mathrm{mg} / \mathrm{day}$, and prednisolone enema, and mesalazine $400 \mathrm{mg}$ three times/day and potassium supplement. She showed considerable improvement in her general well-being and physical ability, notably her foot drop.

At the end of her fourth week in hospital she suddenly collapsed and died. Postmortem examination revealed acute Crohn's disease involving the entire colon and the last $10 \mathrm{~cm}$ of the terminal ileum and a large pulmonary embolus secondary to 
right leg deep vein thrombosis. Postmortem examination did not reveal any other lesions that could have caused or contributed to this patient's peripheral neuropathy.

\section{Discussion}

Crohn's disease and ulcerative colitis are chronic inflammatory bowel diseases that manifest many complications outside the gastrointestinal tract. Among these, nervous system involvement has rarely been noted, despite the presence of predisposing factors such as malabsorption, vasculitis, thromboembolism and amyloidosis.

Peripheral nerve involvement associated with Crohn's disease has been previously described. Duffy et al. ${ }^{1}$ reported a group of children with Crohn's disease who developed a reversible sensory peripheral neuropathy due to metronidazole. Nemni et al..$^{2}$ described two patients who suffered from distal sensory neuropathy with a fluctuating course parallel to the exacerbations of the Crohn's enteritis. Neither of these two cases had vitamin $B_{12}$ deficiency or received metronidazole. A similar fluctuating course of sensori-motor polyneuro-

\section{References}

1. Duffy, L.F., Daum, F., Fisher, S.E. et al. Peripheral neuropathy in Crohn's disease patients treated with metronidazole. Gastroenterology 1985, 88: 681-684.

2. Nemni, R., Fazio, R., Corbo, M., Sessa, M, Comi, G. \& Canal, N. Peripheral neuropathy associated with Crohn's disease. Neurology 1987, 37: 1414-1417.

3. Humbert, P., Monnier, G., Billerey, C., Birgen \& Dupond, J.L. Polyneuropathy: an unusual extraintestinal manifestation of Crohn's disease. Acta Neurol Scand 1989, 80: 301-306.

4. Lossos, A., Argov, Z., Ackerman, Z. \& Abramsky, O. Peripheral neuropathy and folate deficiency as the first sign of Crohn's disease. J Clin Gastroenterol 1991, 13: 445-447.

5. Contamin, F., Ollat, H., Levy, V.G. \& Thierman-Duffaud, D. Atteinte des systemes nerveux peripherique et pyramidal au cours d'une maladie de Crohn: role determinant d'une carence folique. Sem Hop Paris 1983, 59: 1381-1385. pathy was described by Humbert et al. ${ }^{3}$ in a patient with Crohn's disease in whom an immunological mechanism was suggested because of concurrence with cutaneous vasculitis.

Lossos et al. ${ }^{4}$ reported a case of severe sensory peripheral neuropathy associated with low serum folate in whom the diagnosis of Crohn's disease was made 2-3 years later. Cases of peripheral nerve involvement in the form of polyneuropathy and mononeuritis multiplex have also been reported in relation to Crohn's disease. ${ }^{5-8}$

In the present case, we found no evidence of antecedent infection or other disease that might have caused or contributed to the clinical condition. A drug effect is also unlikely as she had not taken any drug that is known to cause peripheral neuropathy. We conclude that the clinical course of our patient supports a possible causal relationship between Crohn's disease and peripheral neuropathy.

\section{Acknowledgement}

Special thanks to Gillian Johnstone for her secretarial support.

6. Rankin, G.B., Watts, H.D., Melnyk, C.S. \& Kelley, M.L. National cooperative Crohn's disease study: extraintestinal manifestation and perianal complications. Gastroenterology 1979, 77: 914-920.

7. Danzi, J.T. Extraintestinal manifestation of idiopathic inflammatory bowel disease. Arch Intern Med 1988, 148: 297-302.

8. Cohen, M.G. \& Webb, J. Brachial neuritis with colitic arthritis. Ann Intern Med 1987, 106: 780-781. 